\title{
Ionospheric conductance distribution and MHD wave structure: observation and model
}

\author{
F. Budnik, M. Stellmacher, K.-H. Glassmeier, S. C. Buchert \\ Institut für Geophysik und Meteorologie, Technische Universität Braunschweig, Germany
}

Received: 30 April 1997 / Revised: 27 July 1997 / Accepted: 29 July 1997

\begin{abstract}
The ionosphere influences magnetohydrodynamic waves in the magnetosphere by damping because of Joule heating and by varying the wave structure itself. There are different eigenvalues and eigensolutions of the three dimensional toroidal wave equation if the height integrated Pedersen conductivity exceeds a critical value, namely the wave conductance of the magnetosphere. As a result a jump in frequency can be observed in ULF pulsation records. This effect mainly occurs in regions with gradients in the Pedersen conductances, as in the auroral oval or the dawn and dusk areas. A pulsation event recorded by the geostationary GOES- 6 satellite is presented. We explain the observed change in frequency as a change in the wave structure while crossing the terminator. Furthermore, selected results of numerical simulations in a dipole magnetosphere with realistic ionospheric conditions are discussed. These are in good agreement with the observational data.
\end{abstract}

Key words Ionosphere - (Ionosphere-magnetosphere interactions) - Magnetospheric physics - Magnetosphere - ionosphere interactions - MHD waves and instabilities.

\section{Introduction}

ULF pulsations have been observed for more than a century and have interpreted following Dungey's work (1954) as magnetohydrodynamic (MHD) waves in the magnetosphere. Current understanding of MHD waves in the magnetosphere is well summarized in the reviews by Samson (1991), Allan and Poulter (1992) and Glassmeier (1995). For MHD modes, especially the

Correspondence to: F. Budnik
Alfven mode, the ionosphere is the most important magnetospheric boundary and their interaction has been examined by many authors (e.g. Hughes, 1974). The day-side ionosphere, especially the E-region is a very good conductor and is therefore expected to be a good reflector for Alfvén waves. However, the night-side ionosphere lacks ionisation by the Sun's radiation. Under these conditions the ionosphere is one of the main damping mechanisms for Alfvén waves in the magnetosphere (Newton et al., 1978; Glassmeier et al., 1984). Furthermore it affects the mode structure of the waves, because with changing boundary conditions the $3 \mathrm{D}$ wave equation has different eigenvalues and hence different eigensolutions for a particular field line. Cummings et al. (1969) derived eigenperiods of the guided toroidal and poloidal wave mode resonances for a range of $L$-values and plasma density distributions assuming a perfectly reflecting ionosphere. Allan and Knox (1979a, b) first suggested the existence of quarter waves in the magnetosphere in which the electric perturbation has a node in one ionosphere and an antinode in the conjugate ionosphere. This is caused by a different sign of the ionospheric reflection coefficients in both hemispheres. Therefore, quarter waves are most likely to be observed around the solstices. Allan (1983) derived eigenvalues similar to Cummings et al. (1969) for quarter waves and, in addition, examined the evidence for observations of quarter wave ULF pulsations. He presented three pulsation events and concluded that only one is likely to be a quarter wave.

The purpose of this study is to apply the well-known ionospheric reflection theory to an observed ULF pulsation event in the dusk sector when crossing the terminator, i.e. when crossing geomagnetic field lines exhibiting different ionospheric reflection conditions. We propose that this observation shows a transformation of a half wave into a quarter wave harmonic toroidal mode. The work is divided in five sections. Next we present the ULF pulsation event and our interpretation. The third section offers a theoretical approach for the case where the ionospheric reflection coefficient 
allows the existence of quarter waves. To verify our interpretation in principle we use results of a numerical code presented in section four. Finally a summary and conclusion is given.

\section{ULF pulsation event of May 3, 1985}

The event is recorded by the magnetometer of the space environment monitor instrument package aboard the Geostationary Operational Environmental Satellite 6 (GOES-6). GOES-6 operates in a geostationary orbit, that is 6.67 Earth radii $\left(R_{E}\right)$ and $0^{\circ}$ latitude, at a longitudinal position of $107.6^{\circ} \mathrm{W}$ in a geocentric geographic coordinate system (GEO). The magnetic field is described by three perpendicular components: $B_{x}$ radially pointing earthward, $B_{y}$ azimuthally pointing eastward and $B_{z}$ perpendicular to the satellite's orbital plane. These, as well as the magnitude $|B|$ are shown in Fig. 1 for the time interval between May 2 1985, 23:30 UT and May 3 1985, 2:10 UT. In the $B_{x}$ and $B_{y}$ component one can recognise a pulsation which changes its frequency and amplitude at around 0:52 UT, that is 17:52 MLT. Beforehand, the amplitude ranges up to $\sim 3.5 \mathrm{nT}$, but afterwards only to $\sim 1.5 \mathrm{nT}$. In the $B_{z}$ component as well as in the magnitude $|B|$ the pulsation is not clearly visible. This indicates a mainly transverse polarized wave.

To distinguish better between the parallel and perpendicular components the data is rotated into a mean field aligned (MFA) coordinate system. The time series are then tapered and the average together with the linear trend are subtracted before they are Fourier transformed. The obvious artificial irregularities in the former $B_{z}$ component may cause errors in the calculated frequencies. This has been tested thoroughly and no effect on the frequency range considered here has been detected. Resulting dynamical spectra with a frequency resolution of $0.65 \mathrm{mHz}$ are shown in Fig. 2. Most of the spectral energy is stored in the transverse component. One can see a continuous pulsation from 23:45-1:00 UT with a frequency around 18-19 $\mathrm{mHz}$. Additionally a pulsation around $15-16 \mathrm{mHz}$ is visible over a short period of time. Afterwards, from 0:45-2:00 UT a pulsation with $5-6 \mathrm{mHz}$ is excited. The frequency changes over more than $10 \mathrm{mHz}$ and it is remarkable that it does not change sharply but the waves seems to overlap in a certain area. Such a gradual change could be an artifact of the data sliding used in the spectrograms, but it can also be seen in the time series (Fig. 1). The parallel component has only a small peak at 5-6 $\mathrm{mHz}$ between 1:20-1:40 UT.

To find the ionospheric conditions we calculated the footpoints of the local field lines at $110 \mathrm{~km}$ altitude, that is the typical height of the ionospheric E-region. This has been done by field line tracing using the International Geomagnetic Reference Field (IGRF) and the Tsyganenko 89 magnetic field model (Tsyganenko, 1989) in hourly steps between May 2, 1985 12:00 UT and May 3, 12:00 UT. The footpoints are given in Fig. 3 for the Northern and Southern Hemisphere. Local time changes of the configuration of the magnetic field cause the variation of the mapped footpoint coordinates. In the Northern Hemisphere they cover the area from $54.6^{\circ} \mathrm{N}$ to $57.4^{\circ} \mathrm{N}$ and $97.2^{\circ} \mathrm{W}$ to $100.5^{\circ} \mathrm{W}$ which is in the central part of Canada. In the Southern Hemisphere they reach from $69.6^{\circ} \mathrm{S}$ to $71.4^{\circ} \mathrm{S}$ and from $146.1^{\circ} \mathrm{W}$ to $150.4^{\circ} \mathrm{W}$, which is close to Antarctica. In corrected geomagnetic coordinates the footpoints are around $66.5^{\circ} \mathrm{N}, 36.2^{\circ} \mathrm{W}$ in the Northern and $66.3^{\circ} \mathrm{S}, 37.5^{\circ} \mathrm{W}$ in the Southern Hemisphere.

The $K_{P}$ index indicating geomagnetic activity is given in Table 1 for the respective time interval. The indices are relatively small and the day is geomagnetically very quiet. For $K_{P} \leq 2$ the auroral oval is restricted in the dusk sector to geomagnetic latitudes greater than $70^{\circ}$ (Hardy et al., 1987). Therefore we can assume that the calculated footpoints are far away from the auroral zone. Hence ionisation by UV-radiation is the main source for the ionospheric electron density and mainly determines ionospheric conductivities. This justifies the use of the International Reference Ionosphere 1990 (IRI90) (Bilitza, 1990; Bilitza et al., 1993). The IRI90 provides electron densities and ion compositions. The neutral gas density is given by the COSPAR International Reference Atmosphere 1986 (CIRA) (Rees, 1988). These parameters allow the calculation of the ionospheric conductivities and the Pedersen conductance. The data and the calculation tools for the conductivities were obtained from the World Data Center (WDC) C2 for Geomagnetism (1996) in Kyoto, Japan. For the calculation the number of sunspots is also needed. The time interval coincides with a sunspot minimum and the number is set to 14/15 (Sunspot Index Data Center, 1996). The integration boundaries were chosen at 90 and $180 \mathrm{~km}$ altitude, following the work by Schlegel (1987). These values include the maximum of Pedersen conductivity which is usually at $110-120 \mathrm{~km}$ altitude.

In Fig. 4 the Pedersen conductance of the ionospheres at the footpoints are plotted versus UT and MLT in the Northern (dashed line) and Southern Hemisphere (solid line). In the Northern Hemisphere the conductances ranges from $0.03 \mathrm{~S}$ at around 0:00 MLT to 5.0 S at 12:00 MLT whereas in the Southern Hemisphere the conductance is constant near $0.03 \mathrm{~S}$ for a long time period and reaches its maximum of $1.1 \mathrm{~S}$ at around 15:00 MLT. The difference in both hemispheres is due to the seasonal dependence of the solar zenith angle. It can be seen that around the time where the frequency step occurs, which is indicated by the dotted line, the Pedersen conductance in the Southern Hemisphere reaches relatively low values.

Reference values can always include some errors that lead to a deviation from real values. Possible errors are listed next:

1. The field line tracing is based on the IGRF and the Tsyganenko 89 model. This may be inaccurate to the order of one degree in latitude and longitude, which will not cause a large difference in the conductances.

2. The IRI90 and the CIRA models may be inaccurate to an order which is difficult to estimate. In particular 


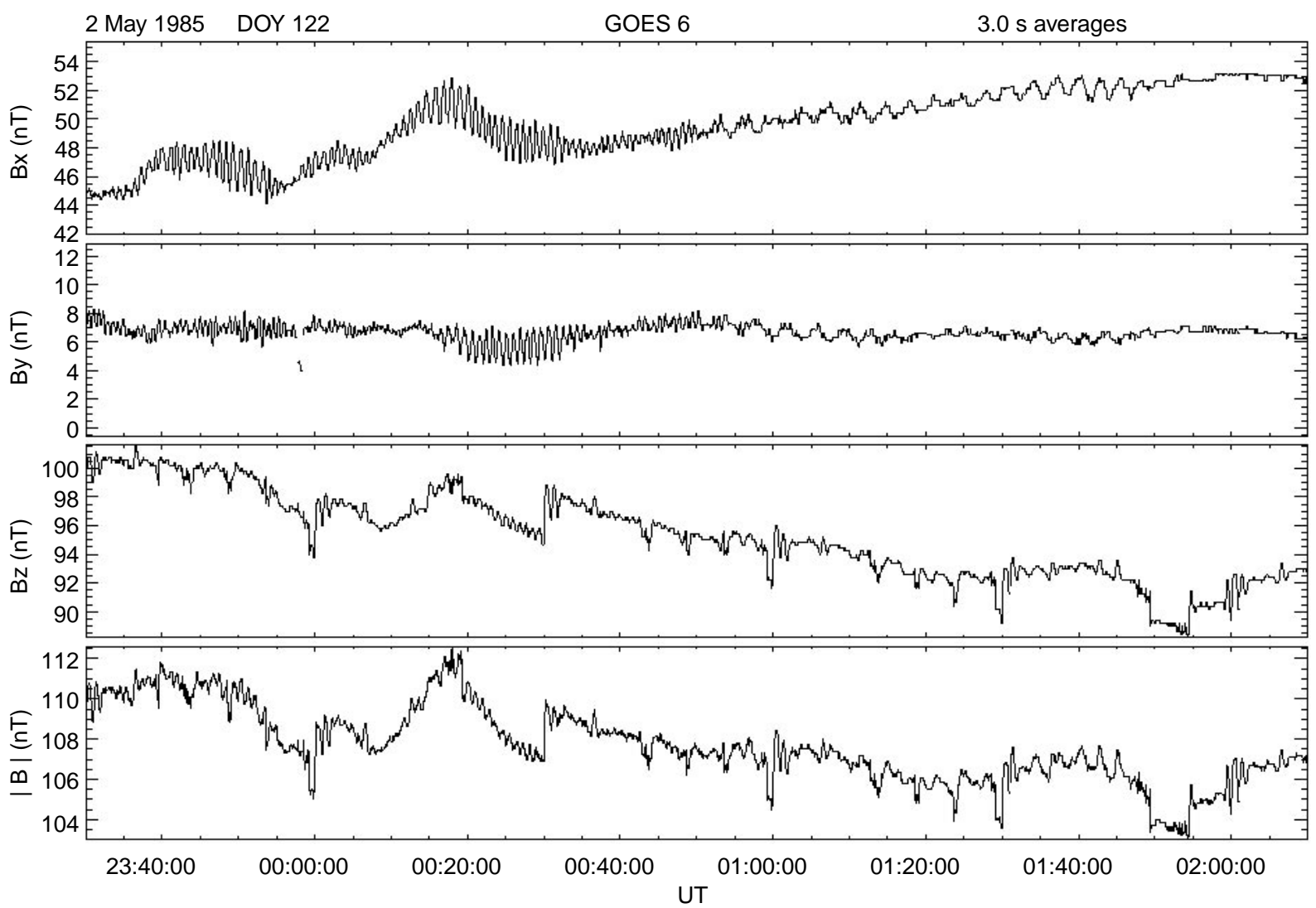

Fig. 1. Time series of the three components and the calculated magnitude of the magnetic field

in the remote regions considered here, where not many observations were made, the reference values can differ from the real values.

3. The choice of the integration boundaries to calculate the conductances is more or less arbitrary. The thickness of the layer where the conductivity contrib- utes significantly to the conductance varies with time and space. Our chosen range seems to be rather thicker than it needs to be.

The overall error is probably less than $10 \%$ for both curves in Fig. 4.

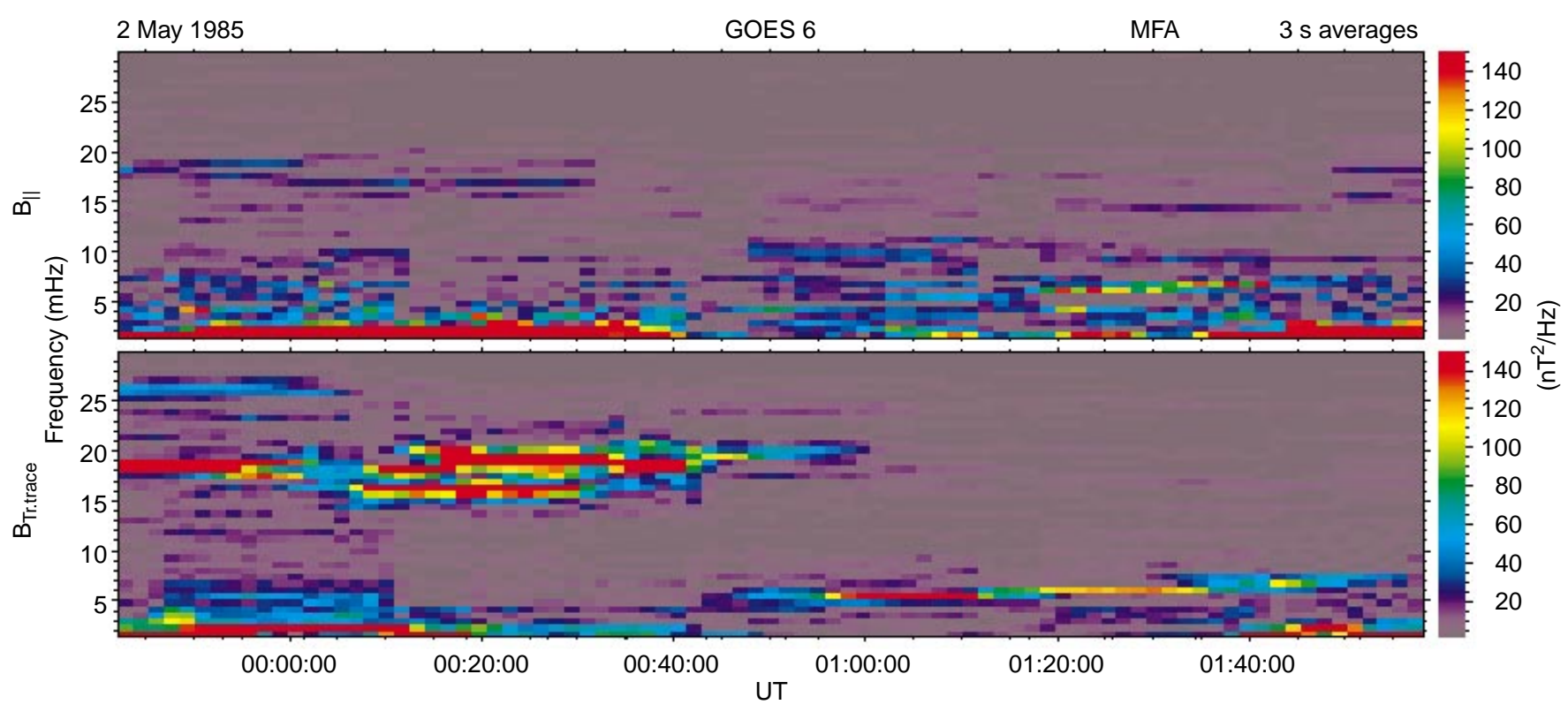

Fig. 2. The dynamic spectra of the parallel and transverse component in a mean field aligned coordinate system 

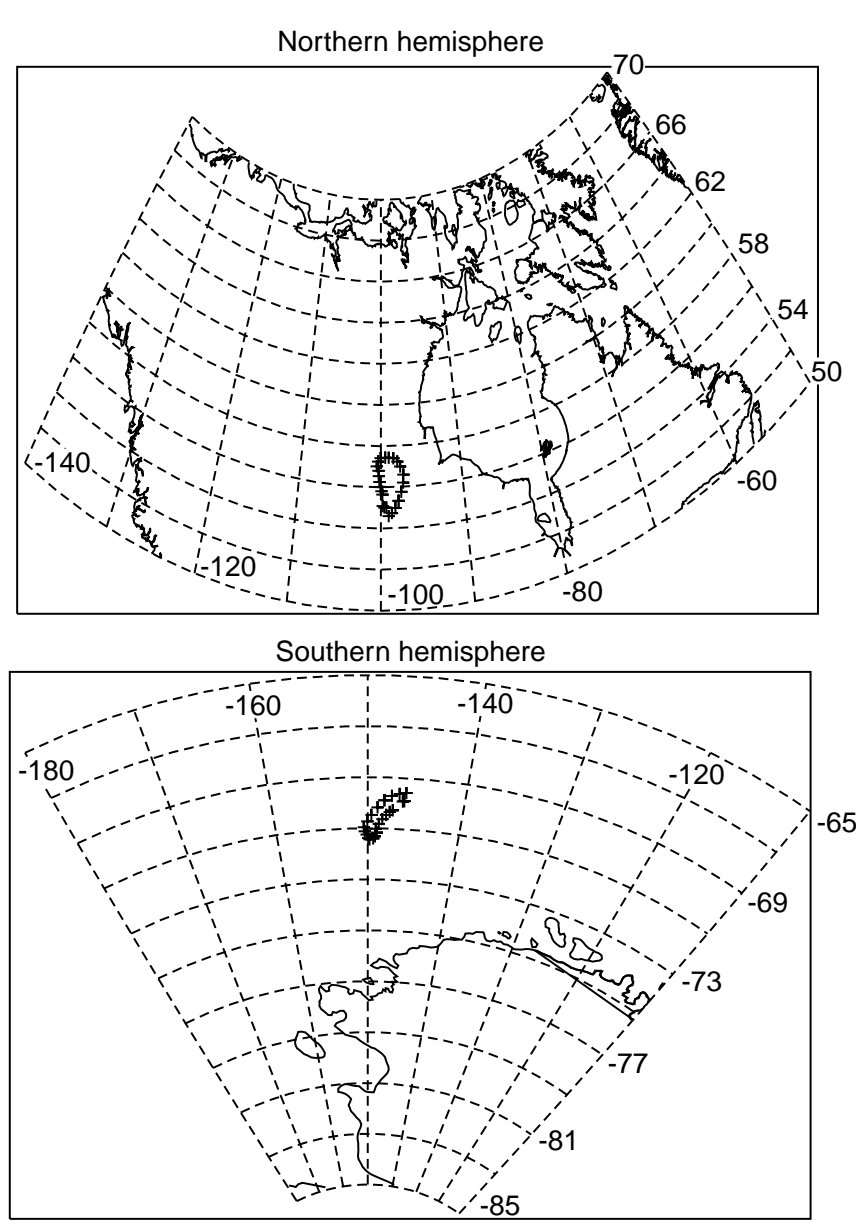

Fig. 3. Footpoints of the field lines in the Northern and Southern Hemisphere. The coordinates are given in a geographic coordinate system

The considerations described in this section indicate a relationship between the day-night terminator and the observed frequency step. In the following we propose a theoretical model to verify the observational features.

\section{Theoretical considerations}

For the purpose of this study the ionosphere can be considered as a thin conducting sheet with height integrated Pedersen and Hall conductivities $\Sigma_{P}$ and $\Sigma_{H}$, respectively. Furthermore, we consider only smooth changes in the spatial distribution of the conductances, e.g. only small gradients, such as the diurnal variations due to the solar zenith angle dependence of the conductances (Brekke and Moen, 1993). Strong gradi-

Table 1. $K_{P}$ indices between May 2, 1985 and May 3, 1985 (WDCC2 Kyoto, 1996)

Time $\quad 12-15 \quad 15-18 \quad 18-21 \quad 21-00 \quad 00-03 \quad 03-06 \quad 06-09 \quad 09-12$ (UT)

\begin{tabular}{lllllllll}
\hline$K_{P}$ & $3-$ & $2-$ & $1+$ & $2-$ & 1 & $1-$ & 2 & $3-$ \\
\hline
\end{tabular}

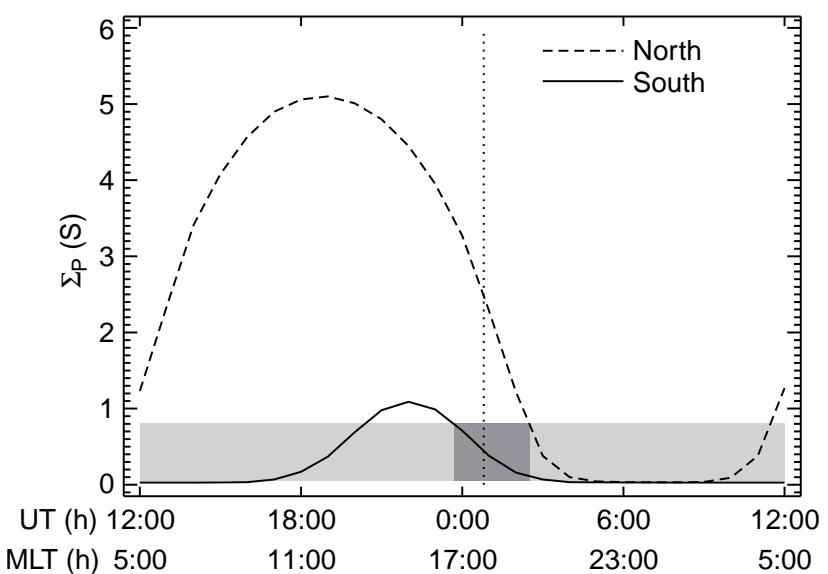

Fig. 4. Pedersen conductance after the IRI 90 at the footpoints in the ionospheres in the Northern (dashed line) and Southern (solid line) Hemisphere. The time interval is May 2, 1985, 12:00 UT May 3, 1985, 12:00 UT. The dotted line indicates the time of the observed frequency step. The light grey shaded region shows the range of typical values of the magnetospheric wave conductance. The dark grey shaded region indicates the occurrence of different signs in the reflection coefficient at conjugate ionospheres

ents, e.g. as caused by particle precipitation, are not included as the GOES-6 fieldline intersects the ionosphere at clearly subauroral latitudes. Therefore gradient effects described in earlier works (Ellis and Southwood, 1983; Glassmeier, 1984) can well be neglected and the reflection process can be described with a scalar reflection coefficient $R$ (Scholer, 1970):

$\vec{E}_{R}=R \vec{E}_{I}=\frac{\Sigma_{A}-\Sigma_{P}}{\Sigma_{A}+\Sigma_{P}} \vec{E}_{I}$

$\vec{E}_{I}$ and $\vec{E}_{R}$ are electric fields of the incident and reflected wave, respectively, and $\Sigma_{A}=1 / \mu_{0} v_{A}$ is the wave conductance of the magnetosphere with the local Alfvén velocity $v_{A}$. This means that the field-aligned current carried by an Alfvén wave is closed by Pedersen currents only. Any Hall current effects are neglected here. Because the Pedersen conductance as well as the wave conductance can vary smoothly in space the reflection coefficient is also a function of space.

Typical values for the Pedersen conductance away from auroral activity are $\sim 4 \mathrm{~S}$ during day-times and $\sim 0.03 \mathrm{~S}$ at night. In the auroral oval it can reach values around $20 \mathrm{~S}$. Furthermore it depends on latitude, season and solar activity. The wave conductance just above the ionosphere has typical values ranging from about $0.05-$ 0.8 S (Allan and Knox, 1979b), which can also be derived from calculated damping constants (Newton et al., 1978, Glassmeier et al., 1984).

From Equation (1) it can be seen that $R$ can have different signs for different values of $\Sigma_{P}$. If $\Sigma_{P}>\Sigma_{A}, R$ is negative and if $\Sigma_{P}<\Sigma_{A}, R$ is positive. This changes the phase relation between the transverse electric field $\vec{E}_{\perp}$ and transverse magnetic field $\vec{b}_{\perp}$ of Alfvén waves, reflected off the ionosphere. For the extreme case $R=-1, \vec{E}_{\perp}$ has a node and $\vec{b}_{\perp}$ an antinode at the ionosphere and vice versa for $R=+1$. When the ionospheres in the Northern and Southern Hemisphere 
have different signs in $R$, quarter waves may appear. Possible mode structures along the geomagnetic field line are displayed in Fig. 5. The first half harmonic with electric nodes in the ionospheres is shown in the top panel, the first quarter harmonic in the middle panel and the first half harmonic with electric antinodes in the ionospheres in the bottom panel. Half waves and quarter waves differ in wavelength and frequency, because the eigenvalues of the toroidal wave equation change with the boundary conditions.

The wave conductance as well as the Pedersen conductance depend on the background conditions like the electron density $n_{e}$ and the ambient magnetic field $B$. If we now consider a very low electron density $n_{e}$, which would result in low Pedersen and wave conductances, the question arises whether $\Sigma_{P}<\Sigma_{A}$ can ever be achieved. We estimate the Pedersen conductance as $\Sigma_{P}=\sigma_{P} \Delta h$ with $\sigma_{P}=\left(n_{e} e / B\right) \cdot \kappa_{i} /\left(1+\kappa_{i}^{2}\right)$ and $\Delta h$ as the thickness of the E-layer. $e$ is the electron charge and $\kappa_{i}$ the ratio of the ion collision to the ion gyrofrequency, which is unity at the maximum conductivity. Taking this maximum the condition $\Sigma_{P}<\Sigma_{A}$ leads to the expression

$n_{e}<\frac{4 m_{i}}{e^{2} \mu_{0} \Delta h^{2}}$

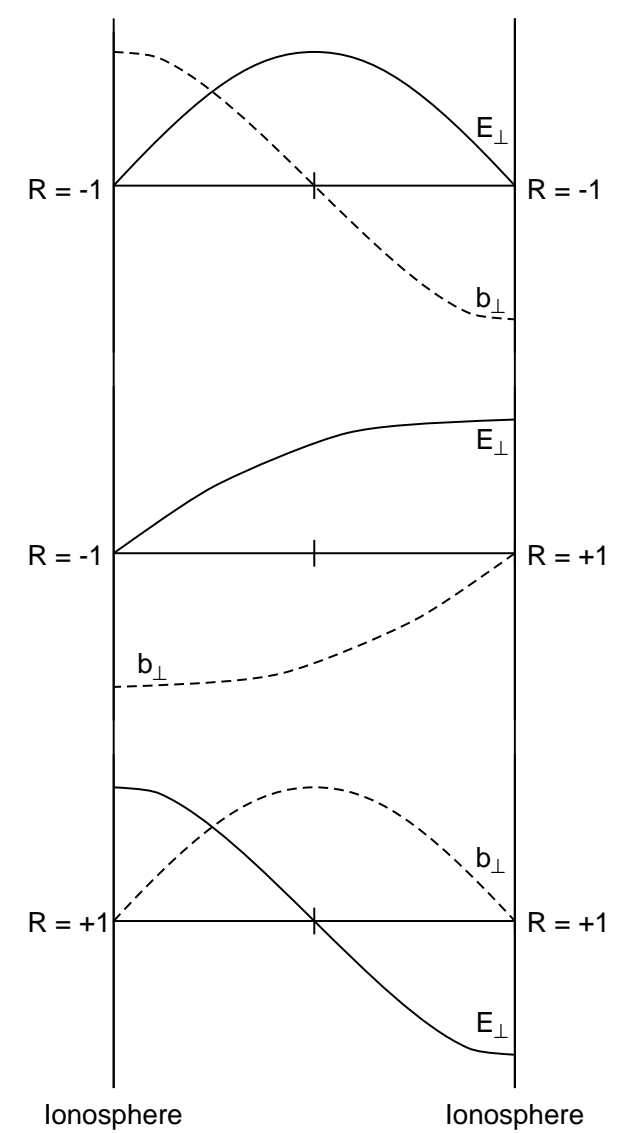

Fig. 5. Three cases of mode structures along the field line for different ionospheric conditions: the first half harmonic with electric nodes in the ionospheres on the top, the first quarter harmonic in the middle and the first half harmonic with electric antinodes in the ionospheres in the bottom where $m_{i}$ is the mass of the ion species. With typical values for $m_{i}=31$ a.m.u. and $\Delta h=20 \mathrm{~km}$ we obtain $n_{e}<1.5 \cdot 10^{10} / \mathrm{m}^{3}$. During winter the nocturnal electron density can be even below $5 \cdot 10^{9} / \mathrm{m}^{3}$ (Turunen et al., 1993). Quantitative estimates of ionisation sources that maintain the night-time E-region performed by Strobel et al. (1980) lead to an electron density of the order of $1-4 \cdot 10^{9} / \mathrm{m}^{3}$. Hence under quiet night-time conditions the Pedersen conductance can fall below the wave conductance.

Because the time interval of the pulsation event described in the previous section is geomagnetically very quiet and the calculated ionospheric footpoints are far away from auroral activity, we do not expect any strong gradients in the conductivities. We therefore consider the ionosphere as a reflection layer with the reflection coefficient (1). We expect after Fig. 4 in the afternoon sector a negative $R$ in both hemispheres. Hence, the observed pulsation can be interpreted as a half wave harmonic with a frequency of $18-19 \mathrm{mHz}$ (see Fig. 2). After crossing the terminator the Pedersen conductance falls below the wave conductance and we expect a region in the night-side magnetosphere where $R$ is positive in the Southern and negative in the Northern Hemisphere (dark grey shaded in Fig. 4). Therefore the observed sudden difference in frequency indicates a change of the mode structure and a quarter wave harmonic with a frequency of $5-6 \mathrm{mHz}$ is observed.

Because of the relatively low frequency the half wave could be the second harmonic. We do not expect to observe the fundamental half wave, because the corresponding magnetic field has a node at the geomagnetic equator (see top panel of Fig. 5). Then the observed quarter wave coincides with the first quarter wave harmonic and would explain the low frequency. The occurrence of such a low frequency under quiet conditions agrees well with a statistical study performed by Cummings et al. (1969). It is difficult to decide on the basis of our data which mode has been observed exactly, because the eigenfrequencies depend on the density distribution along the field lines and in the equatorial plane (Cummings et al., 1969) and are very different under night-time and day-time conditions (Allan and Poulter, 1992).

These theoretical considerations include several simplifications. To apply them to a more realistic magnetospheric geometry and to verify the observational data, a numerical model was developed.

\section{Numerical simulations}

We performed numerical simulations in a $3 \mathrm{D}$ dipole magnetosphere which is an extension of the one developed by Lee and Lysak $(1989,1991)$ and Lysak and Lee (1992). Reference is made to those papers for a more detailed description.

The basic equations of the model are the cold, linearised MHD equations for the perturbed magnetic field $\vec{b}$ and perpendicular components of the electric field $\vec{E}_{\perp}$ : 


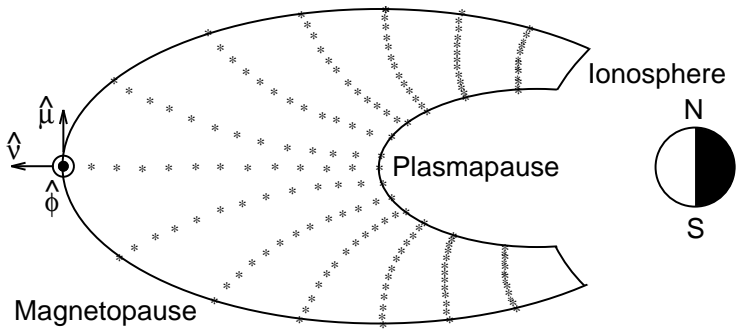

Fig. 6. Boundaries and shape of the dipole model with the dipole coordinate system: $\hat{\mu}$ points parallel to the dipole field, $\hat{v}$ radially outward and $\hat{\phi}$ perpendicular to both in azimuthal direction

$$
\begin{aligned}
(\nabla \times \vec{b})_{\perp} & =\frac{1}{v_{A}^{2}} \frac{\partial \vec{E}_{\perp}}{\partial t} \\
\nabla \times \vec{E}_{\perp} & =-\frac{\partial \vec{b}}{\partial t} .
\end{aligned}
$$

The equations are solved in dipole coordinates $(\hat{\mu}, \hat{v}, \hat{\phi})$ as shown in Fig. 6 by applying finite difference methods in space and time. The magnetosphere is assumed to be axisymmetric with respect to the dipole axis. Boundaries are given by the plasmapause at $L=5(L$ is the McIlwain-parameter), the magnetopause at $L=10$ and the ionosphere at a radial distance of $2 \mathrm{R}_{E}$. Reflecting boundary conditions are used at the plasmapause and the magnetopause, whereas the ionosphere is assumed to have a finite Pedersen conductance by applying:

$\vec{b} \times \hat{\mu}= \pm \mu_{0} \Sigma_{P} \vec{E}_{\perp}$.

This leads with Eq. (4) to the reflection coefficient $R$ in Eq. (1). The sign refers to the ionospheres in the Northern and Southern Hemisphere, respectively. The density profile is the same as in Lee and Lysak (1989), with the density varying with $r^{-6}$ along each field line, and the equatorial density varying with $L^{-3}$. Due to the magnetic dipole field and the inhomogeneous density distribution, the eigenperiods of the field lines have a characteristic form. The first eight harmonics of the half and quarter eigenperiods for each field line are calculated by using the time of flight method (Warner and Orr, 1979) and are displayed versus radial distance in Fig. 7. GOES-6 was at around $L=6.8$ and $9^{\circ}$ latitude during the time of observation. The absolute density is chosen in a way that the frequencies of the fundamental quarter wave and the second half harmonic at $L=6.8$ in Fig. 7 nearly agree with the observed frequencies of the transverse component in Fig. 2. The chosen density distribution then leads to a value of $3.2 \mathrm{a} . \mathrm{m} . \mathrm{u} / \mathrm{cm}^{3}$ at the $\mathrm{s} / \mathrm{c}$ position. This agrees well with densities at geostationary orbit obtained from magnetic and electric field data recorded from GOES-5 and 6 and AMPTE/CCE by Engebretson et al. (1992).

The ionospheric Pedersen conductance in the Southern Hemisphere is fitted with a Gaussian function to the one obtained from the IRI90, which is displayed in Fig. 4 (solid line). For simplicity $\Sigma_{P}$ is chosen to be $3 \mathrm{~S}$ all over the Northern Hemisphere. We now consider the ionospheric footpoints of the geomagnetic fieldlines at

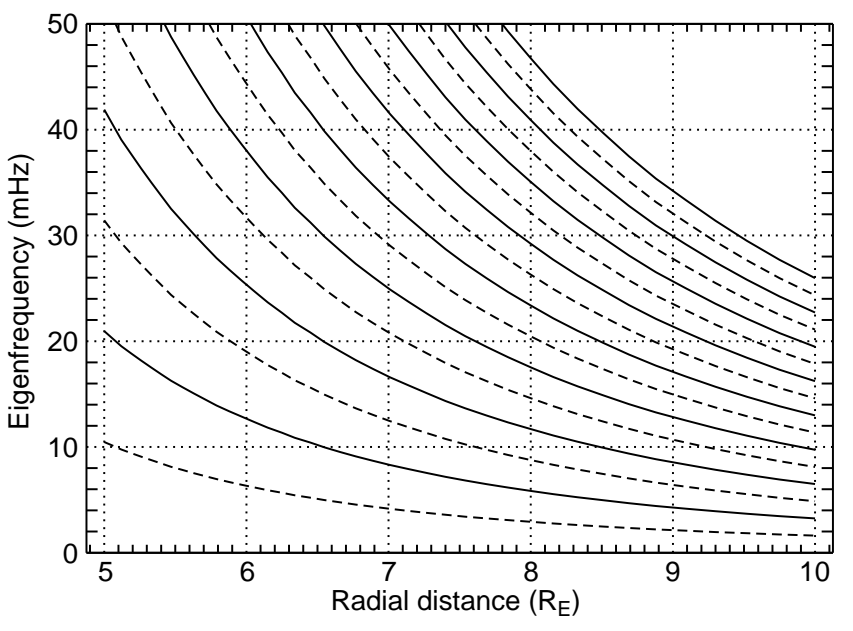

Fig. 7. Eigenperiods of the first eight quarter (dashed lines) and half (solid lines) wave harmonics

the s/c position. There we obtain a wave conductance of $\Sigma_{A}=0.35 \mathrm{~S}$ with the chosen density distribution. If we compare this value with the Pedersen conductance in the Southern Hemisphere (Fig. 4) we find $\Sigma_{P}=\Sigma_{A}$ at about 18:10 MLT. This point of time, when $R$ is changing its sign, is remarkably close to where the frequency step occurs (17:52 MLT).

A local pressure pulse is assumed to be the energy source due to a variation in the solar wind pressure and is applied at the magnetopause at 12:00 MLT. The pulse has the form

$E_{\phi} \sim \exp \left[-\alpha \mu^{2}-\beta \phi^{2}-\gamma t\right] \sin (\omega t)$.

$\alpha$ and $\beta$ are positive constants localising the pulse to approximately $20^{\circ}$ in latitude and $45^{\circ}$ in longitude, respectively. $\gamma$ damps the amplitude of the pulse in time so that it decays after approximately $200 \mathrm{~s}$ simulation time. The frequency of the source is chosen to $v=\omega / 2 \pi=14.3 \mathrm{mHz}$ and lies in between the observed frequencies. The total simulation time is $3600 \mathrm{~s}$ and data are stored every three seconds on a purely azimuthal orbit at the s/c position mentioned above.

The toroidal magnetic field component $b_{\phi}$ is Fourier transformed at each grid point in the azimuthal direction. The spectrum versus magnetic local time is shown as an image plot in Fig. 8. Waves with frequencies about $44.5 \mathrm{mHz}$ and $40 \mathrm{mHz}$ are clearly excited. These are, after Fig. 7, the fifth half and the fifth quarter wave harmonic, respectively. The wave with the higher frequency of $44.5 \mathrm{mHz}$ is mainly visible during daytime between 12:00 and 18:00 MLT whereas the lower frequency of $40 \mathrm{mHz}$ occurs on the night-side between 18:00 and 0:00 MLT. The frequencies do not change sharply but overlap in a certain area as is also seen in the observations (Fig. 2). The terminator is comparable to a kind of a reflection layer. Due to several simplification made in the model there is no possibility of exciting such low frequencies as the observed ones, no matter what excitation frequency is used. But the effect of a abruptly changing frequency after crossing the terminator has been shown in principle. 


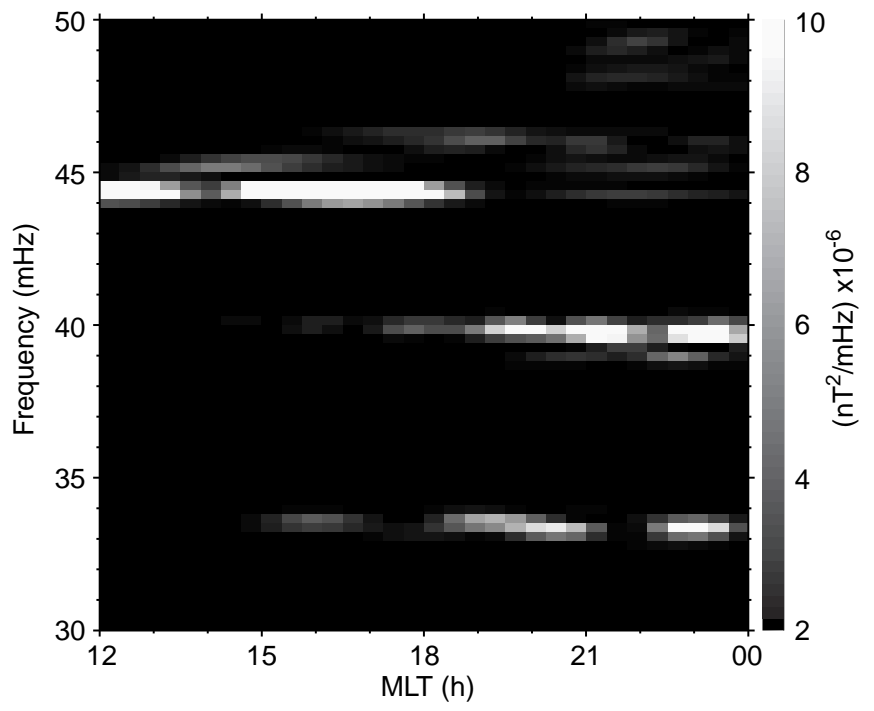

Fig. 8. Fourier-spectrogram of the toroidal magnetic field component $b_{\phi}$ versus magnetic local time at the position of the $\mathrm{s} / \mathrm{c}$ at $L=6.8$ and $9^{\circ}$ geomagnetic latitude

\section{Summary and conclusions}

We presented a pulsation event, observed by GOES-6 during dusk in spring, where the frequency suddenly changes. We propose that this occurs due to a variation of ionospheric conditions. The ionosphere in the Northern Hemisphere has a negative value in $R$ whereas in the Southern Hemisphere it changes from a negative value in the day-side to a positive value when crossing the day-night terminator. We have demonstrated, that this affects the mode structure and the frequencies of the waves. We interpret the jump in frequency as an observation of two different mode structures. The azimuthally moving spacecraft first registered the second half harmonic in the day-side. After crossing the terminator and entering the region with different signs in $R$ for both hemispheres, it recorded the fundamental quarter wave with lower frequency. A modelled magnetospheric density distribution in a dipole magnetic field leads to a plasma density of $3.2 \mathrm{a} . \mathrm{m} . \mathrm{u} / \mathrm{cm}^{3}$ at geostationary orbit which agrees with densities estimated by Engebretson et al. (1992). It is furthermore used to perform numerical simulations with realistic ionospheric conditions. The jump in frequency has been reproduced in principle. The point of time when the jump occurs is in good agreement with the observation.

The likelihood of observing such an event is not very high because it requires waves during very quiet geomagnetic conditions. Allan (1983) interpreted only one of three events presented as a possible quarter wave. This could be caused by different geomagnetic conditions during the three observations. For the event interpreted as a quarter wave the daily sum of the $K_{P^{-}}$ indices was 6 , whereas they were up to 31 and 41 - for the other two observations.

Two kinds of sources seem the most likely drivers of the waves. First the bounce resonance of hot ions.
Waves generated by this mechanism are even harmonics, they occur in the afternoon-evening magnetosphere and they are mostly radially polarised (Hughes et al., 1978), as in the event presented here. The second source could be the Kelvin-Helmholtz instability. The indications for this mechanism are its occurence in the dusk sector and the observation of the geostationary GOES-5 satellite, which moves almost $2 \mathrm{~h}$ earlier in local time. It shows a correlated pulsation at the time ranges concerned. This counts for a more global character of the waves, which is in disagreement with the bounce resonance mechanism. However, both sources provide a broad spectral bandwidth necessary to support two distinct frequencies and are known to last over a long time of order of hours. So it seems that the observed quasi-steady signal can be maintained by energy either from drifting particles or the solar wind flow and is in equilibrium with the energy loss in the ionosphere. These are, of course, only speculations, as there was no further data available for this event.

In future work we will be looking for similar patterns which can confirm our theoretical framework and give additional information about possible mode structures. Furthermore the GOES-5 satellite shows a similar, but not so clear, pattern in its magnetic record which seems to be correlated to the event presented here with some additional features, which we cannot explain yet. This is also subject of further work.

Acknowledgements. The authors are grateful to M. Engebretson for helpful discussions and to the Regionales Rechenzentrum für Niedersachsen, Universität Hannover for providing a lot of computer time. The work by F.B. was financially supported by the German Space Agency DARA. M.S. and K.H.G. were supported through a grant by the German Science Foundation.

Topical Editor D. Alcaydé thanks W. J. Hughes and R. Lysak for their help in evaluating this paper.

\section{References}

Allan, W., Quarter-wave ULF pulsations, Planet. Space Sci., 31, 323-330, 1983.

Allan, W., and F. B. Knox, A dipole field model for axisymmetric Alfvén-waves with finite ionosphere conductivities, Planet. Space Sci., 27, 79-85, 1979a.

Allan, W., and F. B. Knox, The effect of finite ionosphere conductivities on axisymmetric toroidal Alfvén-waves resonances, Planet. Space Sci., 27, 939-950, 1979b.

Allan, W., and E. M. Poulter, ULF waves - their relationship to the Earth's magnetosphere, Rep. Prog. Phys., 55, 533-598, 1992.

Bilitza, D. (Ed.), International Reference Ionosphere 1990, NSSDC 90-22, Greenbelt, Maryland, 1990.

Bilitza, D., K. Rawer, L. Bossy, and T. Gulyaeva, International Reference Ionosphere - past, present, future, Adv.Space Res., 13, 3-23, 1993.

Brekke, A., and J. Moen, Observations of high latitude ionospheric conductances, J. Atmos. Terr. Phys., 55, (11/12), 1493-1512, 1993.

Cummings, W. D., R. J. O'Sullivan, and P. J. Coleman, Standing Alfvén waves in the magnetosphere, J. Geophys. Res., 74, 778793, 1969.

Dungey, J.W., Electrodynamics of the outer atmosphere, Ionos. Res. Lab. Sci. Rep., 69, Penn. State University, 1954. 
Engebretson, M. J., D. L. Murr, K. N. Erickson, R. J. Strangeway, D. M. Klumpar, S. A. Fuselier, L. J. Zanetti, and T. A. Potemra, The spatial extent of radial magnetic pulsation events observed in the dayside near synchronous orbit, J. Geophys. Res., 97, 13741-13758, 1992.

Ellis, P., and D. J. Southwood, Reflection of Alfvén waves by nonuniform ionospheres, Planet. Space Sci., 31, 107-117, 1983.

Glassmeier, K.-H., On the influence of ionospheres with nonuniform conductivity distribution on hydromagnetic waves, J. Geophys., 54, 125, 1984.

Glassmeier, K.-H., ULF Pulsations, in Handbook of Atmospheric Electrodynamics, Ed. H. Volland Vol 2, Ch. 14, CRC Press, 1995.

Glassmeier, K.-H., H. Volpers, and W. Baumjohann, Ionospheric Joule dissipation as a damping mechanism for high latitude ULF pulsations: observational evidence, Planet. Space Sci., 32, 1463-1466, 1984a.

Hardy, D. A., M. S. Gussenhoven, R. Raistrick, and W. J. McNeil, Statistical and functional representations of the pattern of auroral energy flux, number flux, and conductivity, J. Geophys. Res., 92, 12275-12294, 1987.

Hughes, W. J., The effect of the atmosphere and ionosphere on long period micropulsations, Planet. Space Sci., 22, 1157-1172, 1974.

Hughes, W. J., D. J. Southwood, B. Mauk, R. L. McPherron, J. N. Barfield, Alfvén waves generated by an inverted plasma energy distribution, Nature, 275, 43-45, 1978.

Lee, D., and R. L. Lysak, Magnetospheric ULF wave coupling in the dipole model: the impulsive excitation, J. Geophys. Res., 94, (A12), 17097-17103, 1989.

Lee, D., R. L. Lysak, Impulsive excitation of ULF waves in the three-dimensional dipole model: the initial results, J. Geophys. Res., 96, (A3), 3479-3486, 1991
Lysak, R. L., and D. Lee, Response of the dipole magnetosphere to pressure pulses, Geophys. Res. Lett., 19, 937-940, 1992.

Newton, R. S., D. J. Southwood and W. Hughes, Damping of geomagnetic pulsations by the ionosphere, Planet. Space Sci., 26, 201-209, 1978.

Rees, D. (Ed.), Part I: thermosphere model, Adv. Space. Res., 8, 56, 1988.

Samson, J. C., Geomagnetic pulsations and plasma waves in the Earth's magnetosphere, in Ed. J.A. Jacobs, Geomagnetism, Vol 4, 481-592, 1991.

Schlegel, K., Auroral zone E-region conductivities during solar minimum derived from EISCAT data, Ann. Geophysicae, 6, (1), 129-138, 1988.

Scholer, M., On the motion of artificial ion clouds in the magnetosphere, Planet. Space Sci., 18, 977-1007, 1970.

Strobel, D. F., C. B. Opal, and R. R. Meier, Photoionization rates in the night-time E- and F-region ionosphere, Plan. Space Sci., 28, 1027-1033, 1980.

Sunspot Index Data Center, WWW, http://www.oma.be/KSBORB/SIDC/index.html, Brüssel, Belgium, 1996.

Tsyganenko, N.A., A magnetospheric magnetic field model with a warped tail current sheet, Planet. Space Sci., 37, 5, 1989.

Turunen, T., Nygrén, T., Huuskonen, A., Nocturnal high-latitude Eregion in winter during extremely quiet conditions, J. Atmos. Terr. Phys., 55, 783-795, 1993.

Warner, M. R., and D. Orr, Time of flight calculations for high latitude geomagnetic pulsations, Planet. Space. Sci., 27, 679689, 1979.

World Data Center C2 for Geomagnetism, WWW, http:// swdcdb.kugi.kyoto-u.ac.jp/, Kyoto, 1996. 